\title{
Philosophiques
}

\section{Problématique de la preuve en épistémologie contemporaine}

\section{Robert Nadeau}

Volume 7, numéro 2, octobre 1980

URI : https://id.erudit.org/iderudit/203141ar

DOI : https://doi.org/10.7202/203141ar

Aller au sommaire du numéro

Éditeur(s)

Société de philosophie du Québec

ISSN

0316-2923 (imprimé)

1492-1391 (numérique)

Découvrir la revue

Citer cet article

Nadeau, R. (1980). Problématique de la preuve en épistémologie contemporaine. Philosophiques, 7(2), 217-246. https://doi.org/10.7202/203141ar d'utilisation que vous pouvez consulter en ligne.

https://apropos.erudit.org/fr/usagers/politique-dutilisation/ 


\title{
PROBLÉMATIQUE DE LA PREUVE EN ÉPISTÉMOLOGIE CONTEMPORAINE*
}

\author{
par Robert Nadeau
}

On me permettra d'introduire mon propos par une précaution oratoire : la situation dans laquelle je me trouve placé pour vous brosser le tableau de la problématique de la preuve en épistémologie contemporaine est une situation que tout professeur connaît bien et que j'appellerai le dilemme du pédagogue, puisqu'en peu de temps il s'agit de vous expliquer des choses complexes dans un discours que je voudrai jusqu'au bout le plus accessible, sans toutefois tomber dans des simplifications outrancières. Si les choses sont à ce point complexes, c'est d'abord et avant tout parce qu'aujourd'hui, il serait ridicule de prétendre qu'il y a chez les épistémologues consensus sur ce qu'est, ce que peut être ou ce que doit être la science entendue comme activité cognitive. En vous présentant une certaine conception de la théorie scientifique, je ne voudrais donc surtout pas que l'on se méprenne et que l'on pense avoir affaire à une sorte de définition minimale faisant l'unamité au sein de la confrérie : il s'agira plutôt pour moi de vous donner une juste idée de ce qui est en discussion à l'heure actuelle, et non pas de ce sur quoi l'on s'entend jusque-là, et c'est précisément ce que veut dénoter le terme de «problématique »dans le titre choisi ${ }^{l}$.

Mais il y a plus : si les choses sont à ce point complexes maintenant en épistémologie, c'est que la philosophie comme

\footnotetext{
Une première version de ce texte a pu être améliorée grâce aux judicieuses remarques de MM. Claude Panaccio, Normand Lacharité, Claude Savary et François Tournier. Je les remercie sincèrement.

1. Il n'existe pas d'exposition systématique en langue française de l'état actuel de l'épistémologie. Il existe cependant un texte remarquable en langue anglaise dont je me suis très largement inspiré. Il s'agit de Suppe (1974).
} 
discipline a subi depuis environ trois quarts de siècle une formidable mutation. On peut, en un sens, considérer que jusqu'à la fin de l'âge classique, la philosophie englobait encore la science : qu'il me suffise d'évoquer l'attitude exemplaire de Newton qui, en 1687, nous livra ses Principes mathématiques de philosophie naturelle. Or, avec l'avènement du "scientisme " au dix-neuvième siècle, c'est-à-dire de cette attitude à la fois politique et épistémologique qui consiste à prétendre que la science résoudra tous nos problèmes, un renversement s'est en quelque sorte opéré et c'est la science qui est devenue un idéal pour la philosophie, si bien que très rapidement, et dans des écoles de pensée pourtant fort différentes, la philosophie tendit à se considérer d'abord et avant tout comme épistémologie, à savoir comme méthodologie de la science: et elle se voulut elle-même une entreprise rigoureuse, voire scientifique.

Cette mutation formidable peut être caractérisée par l'apparition de deux traits fondamentaux. Le premier peut être vu comme la conséquence de l'émergence, à la fin du dix-neuvième siècle, de ce que nous pourrions appeler le continent langage : en effet, si l'on cherche un premier trait caractéristique de l'épistémologie contemporaine par opposition à la traditionnelle théorie de la connaissance, on le trouve dans le fait que les questions traditionnelles ont été reformulées dans la perspective d'une théorie de la science où la science serait considérée comme un jeu de langage idéal. C'est ainsi que la question épistémologique de base, dont la formulation kantienne était "Que pouvons-nous connaître? », est devenue, aux alentours de $1920^{2}$, la suivante : "Comment est-il possible de formuler adéquatement la connaissance valide que nous pouvons avoir des choses en général ?"

Le second trait caractéristique de l'épistémologie contemporaine, par opposition à la gnoséologie classique ${ }^{3}$, peut être trouvé dans la mise en place d'appareils logiques extrêmement

2. C'est le philosophe allemand Moritz Schlick (1882-1936) qui peut être considéré comme le véritable fondateur de l'épistémologie au sens contemporain du terme, notamment parce qu'il est à l'origine du fameux Cercle de Vienne. Il s'agit en quelque sorte de l'aboutissement du projer de Leibniz.

3. Carnap (1934) considère la gnoséologie comme étant la théorie de la connaissance au sens large, y compris dans sa dimension psychologique, et l'épistémologie comme étant plus spécifiquement la théorie logique de la connaissance scientifique. 
élaborés, ceux de la logistique ou logique algorithmique ${ }^{4}$ : la logique devient ainsi rien moins que le nouveau langage philosophique universel dans lequel il pourra être question de la science, elle-même étant réduite à sa dimension logico-linguistique ${ }^{5}$.

Et nous voilà dans le vif du sujet. Je vous présenterai d'abord ce que j'appellerai le Modèle Épistémologique Standard (M.E.S.) d'une théorie sceintifique en général ${ }^{6}$, pour ensuite vous présenter, dans les deux parties subséquentes de mon exposé, les deux « lieux » de l'épistémologie contemporaine où se trouve formulée la question de la preuve scientifique. Ces deux lieux circonscrits, j'identifierai, dans une quatrième partie, les trois points principaux sur lesquels notre épistémologie s'avère être en crise aujourd'hui. Plan très simple, dont : un modèle, deux lieux d'élaboration théorique et trois points de chute.

\section{Le « Modèle Épistémologique Standard» d'une théorie scientifique}

Tout en simplifiant les choses au maximum, je vous dirai maintenant comment, dans une perspective relativement orthodoxe, l'épistémologie d'aujourd'hui se représente ce qu'il convient de considérer comme une théorie scientifique : pour le dire autrement, le M.E.S. propose à ceux que Gaston Bachelard appelait les "travailleurs de la preuve " un concept de ce qui peut valablement compter comme un langage scientifique. Dans ce modèle, on dénombre cinq composantes

4. L'introduction de la logique formalisée date de Frege (1879); l'usage en fut répandu surtout grâce à Russell \& Whitehead (1910-3).

5. L'épistémologie fait donc de la science un discours de premier niveau dont elle fait la théorie dans un discours de second niveau. C'est à cette relation que nous ferons référence quand nous qualifierons plus loin telle ou telle théorie scientifique de langage-objet et sa reconstruction logique de métalangage. Réduite à sa dimension logico-linguistique, la science est considérée comme un langage utilisant le calcul des propositions et le calcul des prédicats. On aura une bonne idée du fonctionnement de ce langage de premier ordre en consultant, entre autres, les deux seuls ouvrages québécois de logique symbolique, soit Gauthier (1978) et Robert (1978). On considère maintenant que la logique élémentaire est insuffisante pour rendre compte adéquatement du langage de la science.

6. L'expression de Suppe (1974) est "Received View ", qui la reprend de Putnam (1962). Hempel (1970) préfere parler de "Standard Conception " ei Feigl (1970) d' "Orthodox View". 
fondamentales du langage qui peut prétendre servir à formuler une théorie scientifique :

a) ce langage doit pouvoir être considéré comme une "langue bien faite", un langage qui, à cause de ses qualités syntaxiques et sémantiques particulières, nous permettra d'attribuer des propriétés à des objets dans des énoncés que l'on pourra dire vrais ou faux. Peu importe ici comment le domaine des objets en question est circonscrit et de quel type d'objets il s'agit - il peut s'agir, par exemple, de structures grammaticales, de structures moléculaires, de structures de parenté ou d'autres types d'objet - en général une théorie est un discours où se trouve dit ce qu'il en est de ces objets. Dans une discipline donnée, des conflits peuvent bien germer qui concernent en propre la constitution de la classe de ses objets. Par exemple, "La psychologie traite-t-elle de comportements ou d'états mentaux ? " est une question épistémologique importante. Mais quoi qu'il en soit de la réponse à ce genre de questions, il n'en reste pas moins vrai de dire qu'une théorie scientifique est un ensemble d'énoncés bien formés;

b) ce langage comporte trois vocabulaires: celui des termes logiques et mathématiques, où l'on retrouve aussi bien les constantes numériques que les constantes grammaticales comme, par exemple, les conjonctions et prépositions du langage ordinaire (" et ", " ou ", " avant ", " après ", etc.) ; le vocabulaire des termes observationnels, à savoir les termes dont on dit qu'ils désignent directement les êtres dont on fait la théorie (par exemple, "araignée ", "consonne chuintante", "papier de tournesol", «Lune », etc.) ; et enfin le vocabulaire des termes théoriques, à savoir, pour faire bref, tous les autres termes qui ne sont ni des constantes logiques ou mathématiques ni des termes observationnels (par exemple, « arachnide», " phonème ", " réaction chimique ", " orbite elliptique ", etc.) ;

c) les termes du vocabulaire d'observation sont compris comme se référant directement à des objets physiques observables ou à des attributs ou propriétés observables d'objets physiques ;

d) les termes du vocabulaire théorique sont organisés dans des postulats théoriques: si, par exemple, mes termes théoriques sont "énergie", "vitesse de la lumière " et " masse», ces termes ne sont introduits qu'articulés entre eux (comme dans des définitions) et ne doivent, en 
principe, faire appel à aucun autre terme non primitif. Ces postulats théoriques seront considérés tout à l'heure comme les lois fondamentales à partir desquelles une théorie scientifique particulière tente d'avancer une explication quelconque, ou encore comme les axiomes de ladite théorie ;

e) les termes théoriques sont reliés aux termes observationnels par le jeu d'une gamme d'énoncés dans lesquels se trouvent proposées des règles de correspondance ${ }^{7}$. Par exemple, le terme " masse » en vient à correspondre à cela que telle ou telle procédure expérimentale permet de mesurer. Autre exemple : si «électron» est défini comme la particule élémentaire chargée d'électricité négative, c'est qu'une certaine procédure expérimentale (la chambre d'ionisation, par exemple) permet de "voir " qu'un atome a acquis ou perdu un ou plusieurs électrons et est devenu ainsi porteur d'une certaine charge électrique. Il en va de même et il doit en aller de même pour tout terme théorique, comme " phonème " (unité distinctive du sens), "héridité " (processus de transmission chromosomique des caractères spécifiques et individuels), "classe sociale " (unité fonctionnelle d'un ensemble de personnes dans un processus de production économique), etc.

Il est facile de constater qu'au cœur de ce modèle se trouve la nécessaire distinction des termes observationnels et des termes théoriques ${ }^{8}$ : un manque à justifier cette distinction mettrait rapidement en péril notre M.E.S. Or, comme nous allons le voir à l'instant, cette distinction centrale ne va pas de soi. Demandons-nous d'abord ce qui peut compter comme une entité «directement observable »: la réponse habituelle consiste à dire qu'est directement observable une chose ou, mieux encore, un état de choses qui peut être constaté par les

7. Les premiers artisans du modèle épistémologique standard, Carnap notamment, avaient cru possible de relier les termes théoriques ( $T \mathrm{t}$ ) aux termes observationnels ( $\mathrm{To}$ ) par le jeu de ce que l'on avait appelé alors des définitions explicites. La version finale du modèle, que l'on retrouve dans Carnap (1956), parle plutôt d'interprétations partielles des Tt en To par des règles de correspondance qui spécifient les procédures expérimentales par lesquelles la théorie est reliée aux phénomènes observables ou encore aux phénomènes observés (" data "). Le statut épistémologique de ces règles, que Suppes (1967) appelle des " coordinating definitions ", est aujourd'hui considéré comme extrêmement problématique. On consultera à ce propos Suppes (1962) et Schaffner (1969).

8. Il est généralement admis que la possibilité de définir tous les termes théoriques d'une théorie donnée en termes observationnels a été établie par William Craig dans un théorème mathématique. L'argument logico-mathématique complet se trouve dans Craig (1953). On en trouve un exposé non technique dans Craig (1956). Une discussion épistémologique en a été faite par Hempel (1958). 
sens sans recours à un quelconque instrument. Ainsi, « bleu », « chaud », « grand ", « dur », « eau ", « bois ", «fer», «poids» sont des termes ${ }^{9}$ qui sont censés dénoter immédiatement pour un observateur éventuel quelque chose d'aisément constatable : cela veut dire que mis en présence d'un énoncé élémentaire ou atomique ${ }^{10}$ de forme " a est bleu ", l'observateur peut décider sans longue réflexion s'il peut accepter ou refuser l'énoncé en question.

Mais, premier problème, l'observabilité n'est pas particulièrement facile à définir quand l'on se veut précis et rigoureux. La difficulté crôit davantage encore si l'on tient à parler d'observabilité directe ${ }^{11}$. Considérons les cas suivants :

$1^{\circ}$ puis-je soutenir que, quand je me regarde dans un miroir, je m'observe directement?

$2^{\circ}$ dira-t-on du garde qui, du haut de sa tour, observe qu'une épaisse fumée s'échappe de la forêt, qu’il observe directement un feu de forêt?

$3^{\circ}$ quand je prétends observer un avion à réaction volant à très haute altitude, est-ce que jobserve directement un tel avion ou plutôt une tache en mouvement qui, parfois, trace un sillon blanc?

9. Tous les termes susceptibles d'être utilisés dans une description d'observation sont dits dispositionnels : par exemple, "rouge » est un terme dispositionnel dans la mesure où il réfere à la disposition qu'ont certaines choses à refléter une certaine sorte de lumière. Depuis Lewis (1929), beaucoup d'épistémologues pensent que tous les termes, théoriques et/ou observationnels, sont dispositionnels. C'est notamment le cas de Popper (1959).

10. Russell (1918) introduit la notion de proposition atomique dans les termes suivants :

"On peut définir les propositions atomiques négativement comme étant des propositions dont les parties ne sont pas elles-mêmes des propositions, et ne contenant pas les notions "tout " ou "quelque ". Ainsi " ceci est rouge ", "ceci vient avant cela " sont des propositions atomiques (Introduction à la deuxième édition, p. XV)

C'est cependant Wittgenstein (1921) qui a élaboré l'atomisme logique dans toute sa radicalité.

11. La première spécification complète de l'expression " directement observable " nous a été fournie par Carnap (1936-7). Pour le M.E.S., la seule façon d'être rigoureux en philosophie consiste à traduire en langage formel ce qui a été formulé en langage intuitif (qualifié de mode matériel de parler). Voici, à titre d'exemple, comment une telle expression se trouve définie par Carnap en mode formel de parier

"Un prédicat ' $\mathrm{P}$ ' $\mathrm{d}$ 'un langage $\mathrm{L}$ est dit directement observable pour un organisme (par exemple une personne) $\mathrm{N}$ si, pour des arguments appropriés ou convenables, par exemple, 'b', $\mathrm{N}$ est capable, en des circonstances appropriées et après un petit nombre d'observations, de décider d'une phrase complète du type 'P(b)' ou ' $\sim P(b)$ ', c'est-à-dire de confirmer que ' $\mathrm{P}(\mathrm{b})^{\prime}$ ou ' $\sim \mathrm{P}(\mathrm{b})^{\prime}$ est le cas à un degré tel que $\mathrm{N}$ puisse ou bien accepter ou bien refuse 'P(b)' ». 
$4^{\circ}$ et quand je décris un mouvement dans une chambre à bulle, est-ce que je décris une trace (terme observationnel) ou plutôt l'émission d'une particule (terme théorique)?

Quoi qu'il en soit de la possibilité de définir rigoureusement l'observabilité, il est important de voir qu'à cet égard, le M. E. S. n'entend pas avoir de valeur inductive : il n'entend pas être la description fidèle et exacte de la pratique scientifique concrète et réelle, il ne se conçoit jamais lui-même comme une généralisation empirique obtenue après étude de nombreux cas d'espèce. Pour les praticiens de l'épistémologie, cette question de l'observabilité, comme toutes les autres questions proprement épistémologiques, est une question d'ordre logique et non pas d'ordre pratique.

Cela nous amène au second problème : quiconque fréquente un tant soit peu la littérature épistémologique pertinente au débat dont je fais état s'aperçoit vite que ce que les épistémologues entendent par «observable " ne correspond pas (que peu du moins) à ce que les scientifiques nomment ainsi pour leur part. Pour un épistémologue, une température de $20^{\circ} \mathrm{C}$, un poids de $27 \mathrm{~kg}$, une pression atmosphérique de $101,3 \mathrm{kPa}$ parce que, tour à tour, l'observation, et donc la confirmation de l'énoncé, requièrent un thermomètre, une balance, un baromètre, ne sont pas (directement) observables. À la limite, même des grandeurs qui peuvent être établies avec des procédures archisimples, requérant tout au plus l'usage d'une règle graduée, d'une horloge, d'un spectroscope à prisme (les choses se compliquent avec une règle à calcul, une horloge atomique, un spectrographe de masse) ne sont pas comme telles, logiquement parlant, observables : ce que l'on observe, c'est un point marqué sur un instrument donné. Par exemple, un courant électrique n'est jamais observé comme tel, il est plutôt inféré d'observations. À la limite, ce qui se trouve mis en cause, c'est, sinon la distinction même entre termes observationnels et termes théoriques, du moins la possibilité d'un langage observationnel pur, c'est-à-dire théoriquement neutre, puisque le moindre instrument est en lui-même une théorie matérialisée et que très peu d'observations scientifiques sont possibles sans instrumentation préalable. 
Quoi qu'il en soit de la solution de ces deux problèmes, il me paraissait important que l'on vît l'essentiel, à savoir ce vers quoi pointe cette distinction fondamentale mise en force par le M.E.S. Dès lors, on concevra que cette distinction veuille simplement marquer la différence entre un énoncé dont la vérité peut être rapidement décidée (et qui sera conçu différemment comme énoncé de base par Karl Popper et comme énoncé protocolaire par Rudolf Carnap), cette décision étant nécessaire à la formation du consensus intersubjectif des chercheurs, et un énoncé qui ne peut pas l'être parce qu'il incorpore des termes hautement construits et qui ne peut être validé que par le recours à des instruments plus ou moins complexes - ce qui exige, en toute logique, de nombreuses observations préliminaires permettant de conclure que l'instrument utilisé est le bon instrument à utiliser pour observer ce que l'on veut et prétend observer.

Mais une seconde distinction, plus importante encore si l'on considère qu'elle se trouve incorporée au M.E.S. malgré qu'elle ait presque deux cents ans d'âge, concerne les deux grandes classes logiques d'énoncés possibles, à savoir les énoncés analytiques et les énoncés synthétiques ${ }^{12}$. Bien qu'au centre de très vives polémiques, cette distinction, tout comme la première, a le mérite de bien faire apercevoir une différence indéniable, peu importe que l'on n'ait pas encore réussi à en justifier correctement la validité. On dira d'un énoncé qu'il est analytique s'il est vrai en vertu du sens des termes qui s'y trouvent employés. Par exemple, l'énoncé « tous les célibataires sont non mariés » est vrai par définition selon le sens habituel que l'on donne à « tous», «célibataire » et « non marié ». La caractéristique logique d'un tel type d'énoncés, c'est qu'il n'a aucun contenu empirique, qu'il n'est pas informatif en ce sens qu'il ne nous apprend rien sur l'état du monde.

12. Cetre distinction remonte à Kant (1781):

"Ou bien le prédicat B appartient au sujet A comme quelque chose qui est déjà contenu d'une manière cachée dans ce concept; ou bien $B$ est tout à fait en dehors du concept $A$, bien qu'il se trouve cependant en liaison avec lui. Dans le premier cas, i'appelle le jugement analytique ; dans l'autre, synthétique " (Introduction, §IV).

Cette distinction, reformulée par Frege (1884), n'a pas vraiment été remise en question avant Quine (1951). On consultera avec profit la défense et l'illustration qu'en ont faites Grice \& Strawson (1956). 
Par opposition, un énoncé synthétique est non analytique au sens où il ne saurait être vrai ou faux par définition, mais en vertu de sa confrontation avec le monde tel qu'il se trouve, et donc tel qu'il se donne dans les multiples expériences qu'on en peut faire. Fondamentalement, cette distinction sert à différencier deux types d'énoncé dont la modalité doit être absolument tenue pour distincte: seuls les énoncés analytiques peuvent être dits nécessairement vrais (et, à la limite, dans tout monde possible), alors que les énoncés synthétiques peuvent être vrais mais sont contingents (c'est-à-dire que leur valeur de vérité, Vrai ou Faux, dépend de la correspondance entre le monde et ce que l'énoncé dit du monde) ${ }^{13}$. Par exemple, l'énoncé "Maurice Duplessis était célibataire» ne saurait être vrai en vertu du sens des noms faisant ici respectivement office de sujet et de prédicat logiques. Cet énoncé n'est vrai que :

$1^{\circ}$ si " célibataire " veut dire " non marié " pour nous et

$2^{\circ}$ si Maurice Duplessis n'a de fait jamais été marié.

Cet énoncé est clairement une hypothèse au sujet d'un état de choses passé, et il ne saurait être dit vrai que s'il décrit une situation du monde qui fut effectivement le cas.

C'est pour ce modèle de théorie que l'épistémologie contemporaine a proposé un double idéal de preuve scientifique, c'est-à-dire de mise à l'épreuve du discours à prétention de vérité ${ }^{14}$. C'est ainsi que l'on dira d'une théorie qu'elle est prouvée si elle a été, d'une part, confirmée ou corroborée suffisamment par les faits ${ }^{15}$, et si, d'autre part, elle se laisse reconstruire rationnellement suivant les canons de la méthode axiomatique.

\section{La preuve comme corroboration}

La distinction entre énoncés analytiques et synthétiques permet de distinguer les sciences logico-formelles et les

13. Tarski (1944) a élaboré un concept sémantique de vérité qui permet de penser la forme logique du concept de correspondance.

14. Il deviendra clair plus loin que, dans cette entreprise, l'épistémologie est tributaire d'une théorie de la preuve, dite aussi théorie des démonstrations, élaborée par la métamathématique, c'est-à-dire par l'analyse logico-mathématique des fondements des mathématiques.

15. Dans la tradition du positivisme logique, le terme utilisé est confirmation. La notion de corroboration a été introduite par Popper (1959), chapitre X. 
sciences empiriques, et permet aussi de faire le partage entre deux types de propositions dans toute théorie empirique quelle qu'elle soit, à savoir celles qui seront considérées vraies par définition et celles qui ne pourront être tenues pour vraies qu'au terme de leur confirmation par l'effectuation d'une série d'observations. Ce qui permet d'attribuer un statut empirique à une théorie, c'est la présence, au sein du système d'argumentation, d'énoncés à contenu empirique. Ces énoncés peuvent avoir deux formes logiques : on dira de l'énoncé qui porte sur tous les individus d'un ensemble donné qu'il est universel, et de l'énoncé qui ne porte que sur un ou quelques-uns de ces individus qu'il est existentiel ${ }^{16}$. L'énoncé existentiel ou singulier sert à déclarer qu'un événement particulier ${ }^{17}$ se produit, s'est produit ou se produira, alors que l'énoncé universel sert à formuler des régularités régissant sans exception l'ensemble des phénomènes d'un certain type ${ }^{18}$.

Ce sont de tels énoncés universels qui constituent les lois que le scientifique essaie de corroborer. On a dit du M.E.S. qu'il était "explicationniste » justement parce qu'il proposait de n'accepter comme théorie scientifique que les systèmes d'énoncés visant à expliquer un ensemble donné de phénomènes, c'est-à-dire à les subsumer sous une loi ${ }^{19}$. Il est évident cependant qu'une loi universelle de la nature, formulée dans un énoncé universel de forme "Tous les F sont B ", transcende d'emblée toute vérification possible, puisque tous les $\mathrm{F}$ d'un certain type faisant l'objet d'une science donnée ne peuvent jamais être observés comme tels, seul un nombre fini

16. La distinction ici avancée ne concerne que les énoncés généralisés, c'est-à-dire ceux formés de quantificateurs et de variables : "Un " est le quantificateur existentiel, " tous " le quantificateur universel. On peut aussi vouloir integrer à la théorie comme telle un ensemble d'énoncés non généralisés, qui font plutôt place à des constantes individuelles.

17. Popper (1959) définit ingénieusement un événement comme une classe d'occurrences, chaque occurrence se donnant selon ses coordonnées spatio-temporelles propres.

18. De tels énoncés universels peuvent être remplacés par des énoncés statistiques, comme il semble que ce soit le cas maintenant aussi bien dans les sciences physiques que dans les sciences sociales. Il est donc prévu d'avance et accepté que des exceptions se fassent jour.

19. Un des problèmes épistémologiques majeurs depuis la fin du dix-neuvième siècle est lié à l'idée que toutes les sciences n'auraient pas pour but l'explication et que certaines d'entre elles, les sciences humaines et sociales (appelées aussi, par moment, sciences de l'esprit ou encore sciences de la culture), auraient plutôt pour but la comprébension, et seraient donc justiciables d'une analyse épistémologique sui generis. Ce débat a toujours cours. 
d'expérimentations et d'observations étant possible. Néanmoins, une théorie scientifique n'est dite prouvée ou démontrée que si les lois qu'elle formule ont subi l'épreuve des faits, c'est-à-dire si elles ont été testées dans des conditions suffisamment rigoureuses. La rigueur des conditions de test dont il est ici fait mention a trait à la répétabilité du test et à la nécessité d'un contrôle intersubjectif des résultats obtenus. D'où l'on voit que si le cœur de toute théorie scientifique, au terme du M.E.S., est constitué par l'énoncé d'une loi universelle de la nature, la corroboration ou confirmation de cette loi (énoncé universel) implique un consensus dans l'observation (l'acceptation d'un certain nombre d'énoncés existentiels par un groupe de chercheurs) ${ }^{20}$.

Dans la mesure où une théorie scientifique cherche à expliquer un type de phénomènes, une classe d'événements ou un ensemble de faits ${ }^{21}$, elle doit organiser les énoncés qu'elle formule, les universels et les existentiels, selon un ordre hypothético-déductif : pour le M.E.S., une explication n'est scientifiquement acceptable que si elle suit le modele de la loi de couverture $^{22}$, c'est-à-dire si elle se présente comme une argumentation déductive où l'une des prémisses stipule des états de fait (énoncé de conditions initiales dans des propositions existentielles), et l'autre énonce au moins une loi (énoncé d'une régularité dans une proposition universelle conditionnelle de forme : «pour tout $x$, si $x$ est $F$, alors $x$ est $B$ »), et où la conclusion de la déduction se trouve être l'énoncé de l'événement, du fait ou du phénomène que l'on voulait justement

20. Loin que l'explication des phénomènes soit ici la seule à faire sens, l'explication des lois elles-mêmes est aussi recherchée : on dit d'une loi qu'elle est expliquée si on peut la déduire d'une autre loi scientifique tenue pour vraie (ou suffisamment probable). Ainsi expliquée, une loi n'en est que mieux proutuée.

21. L'idée qu'il ne saurait y avoir de connaissance "scientifique" du singulier remonte à Aristore.

22. Hempel a mis au point dès 1948 , en collaboration avec Paul Oppenheim, le "covering-law model"; ce modèle d'explication est appelé modèle Hempel/Oppenheim ou encore modèle $\mathrm{D}-\mathrm{N}$ (pour déductif-nomologique, c'est-à-dire déduction à partir d'une loi servant de prémisse). Les « conditions particulières " dont il est ici fait mention ont été appelées conditions initiales par Popper (1959). On a coutume de considérer que le modèle $\mathrm{D}-\mathrm{N}$ est un modèle causal d'explication, et ce sont ces conditions initiales qui font office de cause rendant compte de l'événement, cette cause agissant sous l'empire d'au moins une loi. Ce modèle est clairement et simplement exposé dans Hempel (1966). On en trouve un exposé plus technique dans Hempel (1965), chap. 10 
expliquer (énoncé d'une proposition existentielle ou singulière). On dit alors des prémisses qu'elles forment l'explanans et de la conclusion qu'elle forme l'explanandum.

Pour le M.E.S., une théorie qui n'explique aucun événement selon ce schéma déductif ne peut prétendre être scientifiquement valable et fondée car elle ne peut être tenue pour empiriquement prouvée. Selon le nombre et la sévérité des tests qu'on lui aura fait passer, on lui reconnaittra un degré plus ou moins grand de confirmation ou de corroboration : et bien qu'une théorie ne soit jamais vérifiable définitivement, les tests qu'elle aura passés avec succès permettront qu'on la dise, dans une certaine mesure, prouvée ${ }^{23}$. L'adoption du modèle de la loi de couverture impose dès lors au cheminement de toute preuve scientifique d'incontournables contraintes, chacune faisant d'ailleurs l'objet d'attentions particulières dans l'épistémologie d'aujourd'hui.

Signalons d'abord l'obligation de procéder par hypothèses : pour expliquer quelque situation que ce soit, nous avons toujours le choix entre plusieurs hypothèses mais il nous faut émettre au moins une hypothèse. Quand deux hypothèses incompatibles se présenteront simultanément et qu'un test s'avérera possible pour les départager, on parlera d'expérience cruciale $^{24}$. Autrement, il y aura conflit entre hypothèses ou théories rivales. Mais rien ne peut être prouvé ou démontré en science s'il n'y a pas, au départ, formulation d'une hypothèse et, par la suite, test de l'hypothèse. Il faudra donc convenir que le discours scientifique ne parle pas de faits bruts et que toute observation est toujours encadrée par une hypothèse ou un système d'hypothèses : l'empirisme naiff n'a plus sa place en épistémologie précisément parce qu'il est généralement admis que la théorie a préséance logique sur les faits qu'elle tente

23. La question de savoir si ce modèle de la loi de couverture doit réglementer toute explication scientifique digne de ce nom constitue sans aucun doute l'une des principales controverses épistémologiques des dernières années.

24. Lidée d'" experimentum crucis " remonte à Francis Bacon (Notum Organum : 1620), à qui Kant dédia sa Critique de la Raison Pure. Lexpérience que le physicien français Léon Foucault fit en 1850 pour "confirmer définitivement " la théorie ondulatoire d'Augustin Fresnel est un bel exemple de ce qui voulait être une expérience cruciale. Duhem (1906) en rejeta la possibilité même et Popper (1959) tenta de rétablir la crédibilité épistémologique de ce concept. 
d'expliquer ${ }^{25}$. Mais il faudra convenir également que, pour un domaine d'objets donné, toutes les théories rivales sont comparables entre elles, soit parce que les termes et concepts qu'elles utilisent sont sémantiquement invariants, soit parce qu'ils sont réciproquement intertraduisibles ${ }^{26}$.

Ensuite, ce que l'hypothèse centrale d'une théorie scientifique énonce est présumé être une loi de la nature. Or, nous l'avons vu, l'énoncé d'une loi est logiquement invérifiable comme tel puisqu'il transcende toujours le nombre fini d'observations qu'on aura pu faire pour en éprouver le bien-fondé. Il faudra donc convenir que la vérifiabilité n'est ni le critère de signification ni le critère de démarcation des énoncés scientifiques. Mais alors, si les énoncés scientifiques ne sont jamais susceptibles de vérification au sens fort du terme, il faudra comprendre autrement leur prétention à la validité, soit en termes de testabilité, de falsifiabilité ou de réfutabilité. Deux conséquences majeures se font immédiatement jour: dans la mesure où la plupart des théories scientifiques procèdent maintenant à l'énoncé de lois statistiques, il nous faut contester la testabilité des hypothèses probabilistes, car le moins que l'on puisse dire est qu'elle n'est pas évidente. Mais puisque nos théories scientifiques sont invérifiables, qu'elles peuvent donc être indéfiniment remises en question, il nous est loisible de les considérer elles-mêmes comme plus ou moins probables, ce qui explique que l'épistémologie contemporaine ait eu à scruter aussi bien le concept de probabilité fréquentielle que celui de probabilité logique ${ }^{27}$.

Troisièmement, toute hypothèse scientifique, tout énoncé d'une loi de la nature, quelle qu'elle soit, n'est testable que si l'on accepte au départ la vérité d'autres énoncés. Pour parler dans les termes du modèle de la loi de couverture, une loi ne peut être soumise à des tests que si les énoncés auxiliaires stipulant les conditions initiales sont acceptés comme vrais. Or, il se trouve que de tels énoncés prétendent être issus de

25. C'est pourquoi l'on dit de toute observation scientifique qu'elle est nécessairement "theory-laden ", marquée au coin d'une théorie.

26. Le problème logique de la traduction a été posé par Quine (1951) et surtout (1960), celui de l'incommensurabilité sémantique par Kuhn (1962).

27. L'ouvrage classique sur cette question est Carnap (1950). Sur l'irréfutabilité des énoncés statistiques, cf. Popper (1959), chapitre VIII. 
constatations qui ne peuvent jamais être placées elles-mêmes au-dessus de tout soupçon. Il se trouve également que l'énoncé de l'événement qu'une théorie prétend expliquer, tout comme les énoncés de conditions initiales, requièrent la plupart du temps que l'on recoure à des instruments qui ne sont valides que si la théorie suivant laquelle ils sont construits est elle-même vraie. Il faudra donc convenir qu'une hypothèse scientifique isolée n'est jamais testable et que ce que teste l'expérience est un système d'énoncés théoriques, les uns étant présupposés par rapport à d'autres. Il faudra donc convenir qu'a priori un contre-exemple expérimental ne réfute jamais une hypothèse particulière mais qu'elle réfute tout un ensemble d'hypothèses et que l'ajout d'bypothèses ad boc peut toujours servir à rétablir une théorie dans ses prétentions. Il faudra convenir enfin que, si une hypothèse scientifique n'est jamais isolée lorsque mise à l'épreuve des faits, seule l'émission d'une hypothèse supplémentaire permettra de savoir lequel des nombreux éléments théoriques associés à l'hypothèse de départ a été pris en défaut lorsqu'un insuccès se sera présenté dans l'expérimentation. C'est au prix de tâtonnements nombreux, au cours desquels il en va à chaque fois d'un ensemble composite d'hypothèses, qu'une théorie scientifique pourra se voir confirmer ${ }^{28}$.

Quatrièmement, l'adoption du modèle de la loi de couverture nous force à assimiler explication et prédiction ${ }^{29}$. En effet, l'événement que l'on veut expliquer est ou bien antérieur ou bien postérieur dans le temps à l'énoncé de l'hypothèse qui le concerne. Dans un cas comme dans l'autre, dire que l'événement est expliqué, c'est dire qu'une connaissance suffisante des conditions initiales permet (ou aurait permis) que l'on prédise son occurrence sur la base d'une hypothèse donnée : et une hypothèse n'est scientifiquement prouvée que si elle explique quelque chose de cette façon. L'on conviendra donc que l'explication déductive des phénomènes

28. La thèse de l'invérifiabilité et de la non-falsifiabilité d'une hypothèse isolée en théorie physique est due à Duhem (1906); elle a été généralisée pour toute hypothèse par Quine (1951). C'est pourquoi elle est souvent appelée la thèse Duhem-Quine. Les principaux textes afférents à cette problématique ont été réédités par Harding (1976).

29. Cette équivalence a été d'abord posée par Popper (1944-5), puis approfondie par Hempel/Oppenheim (1948). Cf. Hempel (1965), chap. 10. 
est liée à la fonction prédictive de nos hypothèses, ce qui se trouve à rendre compte du même coup du contrôle technologique que la science rend possible. L'on conviendra également ou bien qu'il faut exiger de toute science théorique qu'elle procède de la sorte, ou bien qu'il faut renoncer à reconnâtre la scientificité d'entreprises qui n'admettent pas ce modèle, ou bien qu'il faut se mettre en quête d'autres modèles épistémologiques qui puissent s'avérer tout autant acceptables $^{30}$.

\section{La preuve comme axiomatisation}

Il est un second volet de la problématique de la preuve en épistémologie contemporaine : une théorie scientifique n'est considérée prouvée que si on en a démontré la consistance interne, c'est-à-dire l'absence de contradiction. On peut même affirmer que cette requête épistémologique prévaut contre la première puisqu'il faut ici subordonner la testabilité d'une théorie à sa cohérence : en effet, une théorie qui n'est pas exempte de contradiction ne saurait avoir de prétention à la vérité. Sur un plan strictement logique, un énoncé n'est vrai qu'en vertu de sa forme: on appelle tautologie un énoncé logiquement vrai, c'est-à-dire un énoncé qui est vrai de toute nécessité en vertu de sa structure interne ${ }^{31}$. C'est aussi en vertu de sa forme logique qu'un énoncé peut être déclaré logiquement faux, auquel cas il s'agit d'une contradiction. Or, s'il faut éliminer des théories scientifiques les énoncés autocontradictoires ou incompatibles entre eux, c'est non seulement pour s'assurer de la vérité logique des propositions qu'elles contiennent, mais aussi parce que d'une proposition fausse l'on peut déduire n'importe quoi ${ }^{32}$, ce qui revient à dire qu'une théorie inconsistante ou incohérente ne saurait avoir aucune prétention à la vérité.

30. Au cour de ce débat, on trouve encore lopposition traditionnellement faite entre sciences de la nature et sciences humaines ou sociales. Le débat le plus intéressant a concerné l'histoire, Hempel (1942) soutenant qu'elle se devait d'expliquer les événements par le recours à des "lois générales", Popper (1944-5) soutenant, pour sa part, qu'elle n'était pas une science théorique qui devait formuler des lois universelles comme des lois de tendance par exemple, et Dray (1957) prétendant qu'il était non seulement tout à fait acceptable, mais en fait fondamental que l'histoire ne tombe pas sous le coup du modèle de la loi de couverture.

31. Le paradigme de la tautologie est " $\mathrm{P}$ ou non- $\mathrm{P}$ " : cette assertion est nécessairement vraie.

32. Les médiévaux disaient déjà que "E falso sequitur quodlibet " 
Pour assurer la consistance d'une théorie, le M.E.S. a mis de l'avant la procédure de la reconstruction axiomatique : en vertu de cette procédure, il doit être possible de démontrer que toute assertion d'une théorie scientifique donnée constitue soit un axiome, soit un théorème d'un système logistique ou déductif. La démarche est, à toute fin pratique, fort simple à comprendre bien qu'elle soit le plus souvent très difficile à entreprendre. L'axiomatisation nécessite une symbolisation, c'est-à-dire une opération par laquelle on substituera aux termes désignant les notions constitutives d'une théorie donnée des symboles choisis par convention et qui n'auront que le seul sens que les énoncés postulatoires ou axiomes du système considéré leur conféreront : cette opération vise à éliminer de la théorie en question les significations intuitives dont les termes utilisés sont chargés, de manière à mieux faire apparaitre le schéma logique abstrait construit par ladite théorie. L'axiomatisation consiste ainsi à reformuler une théorie scientifique quelconque en des propositions premières où se trouvent énoncées en langage symbolique les relations logiques posées entre les termes premiers de ce système déductif. Sous réserve de leur compatibilité mutuelle, ces propositions sont avancées à titre d'hypothèses : la reformulation symbolique n'équivaut jamais à la démonstration empirique et ne fait qu'exiger de nous de ne jamais introduire un terme nouveau qui ne soit correctement défini à l'aide de termes premiers, et de ne jamais accepter de proposition nouvelle (théorème) qui ne soit démontrée à l'aide des propositions premières (axiomes ou postulats). L'axiomatisation exige également une formalisation, c'est-à-dire une seconde opération par laquelle les règles mêmes de la définition des termes et de la démonstration des propositions se trouvent convenues sans la moindre ambiguité : il faut donc faire pour les règles logiques suivant lesquelles le raisonnement s'effectue ce qu'il faut faire pour les axiomes à partir desquels il s'élabore, à savoir les énoncer explicitement et exhaustivement. Ces règles sont elles aussi proposées par convention et chacun est libre de poser celles qu'il voudra pourvu qu'il dise quelles elles sont et que, les ayant adoptées, il les suive.

Évidemment, on comprendra qu'un tel système logistique ne peut pas ne pas comporter de symboles non définis, 
puisqu'il faut toujours compter avec quelque chose au départ : les notions premières deviennent les symboles primitifs du système $^{33}$. C'est à ce prix qu'il est possible de réécrire une théorie de manière à passer des formulations plus ou moins intuitives qu'elle contient à des formulations rigoureuses. Axiomatiser une théorie revient ainsi tout simplement à en isoler les idées principales ou les concepts de base, à les organiser entre eux en des énoncés fondamentaux de manière à pouvoir dériver l'ensemble des autres énoncés inhérents à la théorie scientifique concernée. Il est facile de voir que si les termes primitifs du système déductif sont organisés en axiomes, ces axiomes sont nécessairement affirmés sans preuve à l'appui et qu'ils sont considérés comme indémontrables $^{34}$ : le M.E.S. distingue donc nettement une preuve de vérité empirique et une preuve de validité formelle. La validité formelle d'une théorie scientifique donnée tient à ce que, combinés entre eux ou aux autres théorèmes démontrés dans le système déductif, les axiomes doivent permettre, à l'aide d'une stricte procédure de décision, basée sur les seules règles logiques d'inférence, la déduction de tous les théorèmes et des seuls théorèmes de la théorie concernée ${ }^{35}$.

En somme, pour une théorie scientifique quelle qu'elle soit, l'axiomatisation, loin d'être un lit de Procuste, se veut une quête de clarté sinon un gage de probité. Et si cette procédure de décision permet d'éliminer de toute théorie la confusion et l'ambiguité, c'est qu'ici et ici seulement une démonstration définitive est possible. En effet, au sens métamathématique du terme, une preuve n'est possible que

33. John von Neumann les ramène à cinq types : les constantes, les variables, les connecteurs (par ex. les connecteurs de conjonction, de disjonction, de négation), les opérateurs (par ex. les quantificateurs universel et existentiel) et les parenthèses (ou autres symboles fonctionnellement assimilés comme les crochets et les accolades).

34. L'émergence des géométries non euclidiennes au dix-neuvième siècle, dans la mesure où elle fut fondée sur le remplacement du cinquième postulat d'Euclide par d'autres postulats, nous a sans doute amenés à ne plus exiger des axiomes qu'ils soient évidents par eux-mêmes.

35. La métalogique est la théorie des propriétés formelles de tels systèmes logistiques; au sens de cette théorie, un système formel d'énoncés est considéré comme idéalement axiomatisé s'il a été démontré qu'il est consistant (exempt de contradiction), indépendant (aucun des axiomes ne doit s'avérer déductible des autres axiomes), complet (les axiomes sont suffisants pour la déduction des vérités logiques), catégorique (toutes les interprétations du système formel doivent être isomorphes) et décidable (pour tout énoncé qui n'est pas un axiome du système, on doit pouvoir dire s'il s'agit ou non d'un théorème de ce système). 
dans un langage construit comme un système logistique ou déductif et elle prend la forme d'une séquence finie de formules bien construites (axiomes ou théorèmes), cette séquence se terminant également par une formule bien construite mais qui avait été posée au départ comme devant être démontrée. Loin qu'une telle preuve rende une théorie scientifique intangible ou inviolable et fasse disparaitre la nécessité d'abord posée de devoir la confronter aux faits, tout au contraire, l'axiomatisation permet d'exercer un regard critique sur toute théorie qui se prétend empiriquement fondée dans la mesure où elle force l'élimination de toutes les obscurités d'ordre conceptuel qui peuvent y être contenues, et donc qu'elle permet d'apercevoir plus aisément laquelle des nombreuses hypothèses implicitement ou explicitement retenues il faudra peut-être changer pour obtenir de meilleurs résultats expérimentaux ${ }^{36}$.

Si l'axiomatisation constitue le second idéal de preuve d'une théorie scientifique mis de l'avant par le M.E.S., cela ne veut pas dire, tant s'en faut, que la procédure va de soi. La méthode axiomatique ouvre par elle-même un champ de problèmes à l'épistémologie contemporaine, ne serait-ce que parce qu'aucun instrument fondationnel ne saurait s'accorder à lui-même ce que l'on pourrait appeler l'immunité épistémologique. Ensuite, il faut convenir que plusieurs axiomatisations, les unes plus simples ou plus économiques que d'autres, d'une même théorie scientifique sont possibles : elles peuvent bien être logiquement équivalentes mais avoir des vertus épistémologiques diverses. Mais mises à part ces questions de régie interne, l'épistémologie contemporaine est confrontée à trois problèmes fondamentaux afférents à la requête d'axiomatisation comprise comme reconstruction rationnelle.

36. L'axiomatisation des théories scientifiques requiert, cela doit être clair maintenant, tous les recours de la logique et des mathématiques, ce qui explique que ces deuź cisciplines, fondamentales s'il en est, se soient elles-mêmes axiomatisées les premières.

C'est grâce à Frege (1879) puis à Russell \& Whitehead (1910-3) que la logique s'est axiomatisée. Les mathématiques se sont axiomatisées surtout grâce aux travaux de Peano, pour la théorie des nombres naturels, Hilbert pour la géométrie euclidienne, et Zermelo \& Fraenkel pour la théorie des ensembles. L'exemple classique d'axiomatisation d'une théorie empirique a été fourni par Reichenbach (1924) et concerne la théorie de la relativité. Pour une bonne introduction à la problématique épistémologique de l'axiomatisation, on consultera Blanché (1955). 
Le premier problème concerne la nécessité d'axiomatiser nos théories scientifiques. Si, pour certains, l'opération est capitale et d'extrême importance, pour d'autres, la procédure a conquis ses titres logiques mais n'a pas fait ses preuves sur le plan strictement philosophique ${ }^{37}$. Chose certaine, l'axiomatisation n'entend pas occuper toute la place épistémologique et elle ne vise qu'à permettre d'expliciter les structures logiques de la théorie prise pour objet d'enquête. On a raison de prétendre, par exemple, que Peano a clarifié l'arithmétique en montrant que le système des nombres naturels pouvait être entièrement dérivé de cinq postulats ${ }^{38}$. On a sans doute raison également de penser que von Neumann, en décrivant les Fondements mathématiques de la mécanique quantique, en 1932, a produit d'importants résultats scientifiques puisque son axiomatisation de la théorie des quanta a permis de prouver l'équivalence de la mécanique ondulatoire et de la mécanique matricielle. Argumenter en faveur de la nécessité de l'axiomatisation, c'est argumenter en faveur de son utilité, puisqu'il s'agit d'une opération démonstrative extrêmement puissante: dans un tel langage, si toutes les formules sont bien formées, et pourvu que votre interlocuteur les comprenne, vous avez toutes les chances de le convaincre.

Le second problème concerne la possibilité d'axiomatiser nos théories scientifiques. Quoi qu'il en soit de la valeur de cette requête épistémologique, il ne faut pas passer sous silence le fait que très peu de théories scientifiques ont passé le test à ce jour et que ne semblent axiomatisables, au sens fort et précis du terme, que les seuls secteurs disciplinaires hautement mathématisés (arithmétique, géométrie, théorie des probabilités, mécanique classique des particules et des corps rigides,

37. Par exemple, pour Suppes (1968) et Kyburg (1968), il s'agit d'une opération essentielle, alors que Hempel (1970) en est venu à penser quelle lâtsse en plan les principaux problèmes philosophiques.

38. Les cinq postulats de Peano sont :

1. 0 est un nombre ;

2. le successeur de n'importe quel nombre est lui-même un nombre ;

3. deux nombres ne peuvent avoir le même successeur ;

4. 0 n'est le successeur d'aucun nombre;

5. route propriété de 0 appartenant également au successeur de n'importe quel nombre ayant ladite propriété, appartient à tous les nombres.

Dans ce système axiomatique, "le successeur de " est un terme primitif, de même que «zéro » et « nombre». 
mécanique relativiste, etc.) On ne peut évidemment soutenir a priori qu'une théorie scientifique non encore axiomatisée est en elle-même non axiomatisable. Une théorie ne peut être déclarée, comme telle, non axiomatisable que si l'état de développement dans lequel elle se trouve ne rend pas l'opération possible : ne nous cachons pas que la grande majorité de nos théories scientifiques en anthropologie, sociologie, paléontologie, microbiologie, psychologie, linguistique, etc., sont non axiomatisables parce qu'elles ne se laissent pas reconstruire sous une forme déductive ${ }^{39}$. Cette situation étant ce qu'elle est, faut-il y voir une critique du Modèle Épistémologique Standard lui-même, qui exigerait trop des théoriciens, ou plutôt une critique de l'état déplorable dans lequel se trouvent la plupart de nos théories prétendant à la validité scientifique? La question mérite certainement d'être soulevée.

Ne doutons pas, cependant, que c'est à eux-mêmes que les épistémologues adressent les plus sévères critiques : c'est pourquoi le troisième problème concerne la pertinence même de l'axiomatisation. Cette opération, nous l'avons vu, se veut avant tout une reconstruction d'un point de vue syntaxique, c'est-à-dire concernant surtout les relations entre termes et entre propositions. D'où l'accusation portée contre le M.E.S. de ne faire que peu de place aux préoccupations d'ordre sémantique et pragmatique. Ceux qui veulent accorder la priorité à la structure sémantique des théories scientifiques favorisent une stratégie alternative qui fait place aussi aux interprétations possibles des théories, l'interprétation constituant un modèle précisant les conditions de vérité d'une théoric et non plus exclusivement ce qui apparaît en contrepartie comme

39. Pourraient être considérées non axiomatisables, par exemple, des théories scientifiques aussi différentes que :

a) la théorie du système nerveux central de Hebb ;

b) la théorie de l'évolution de Darwin ;

c) la théorie de l'origine de l'univers de Hoyle ;

d) la métapsychologie de Freud ;

e) la théorie de la dérive continentale de Wegener ;

f) la théorie de l'origine de la vie sur l'ĥle de Pâques de Heyerdahl ;

g) la théorie du Big Bang de Penzias et Wilson;

h) la théorie des mutations consonantiques d'Osthoff et Brugmann ;

i) la théorie du matérialisme historique et dialectique de Marx et Engels ;

j) la théorie des pulsars de Hewish ;

k) la théorie de l'hérédité de Mendel, De Vries, Correns et von Tschermak ;

l) la théorie du stress de Selye. 
n'en étant plus que les conditions de cohésion. Ceux qui veulent accorder la priorité à la structure pragmatique des théories scientifiques exigent autre chose. Cette vague "épistémologique » des vingt dernières années ou bien n'accepte pas de dissocier ce que le M.E.S. a distingué, à savoir la question des conditions d'acceptabilité d'une théorie scientifique et celle de ses conditions d'apparition historique, ou bien n'accepte pas que le contexte de découverte d'une théorie scientifique ne puisse faire l'objet d'une analyse logique au même titre que son contexte de justification ${ }^{40}$.

Concernant cette dernière critique, voyons bien que quand les épistémologues ont proposé la méthode axiomatique, ils ont voulu miser sur la valeur logique de la reconstruction, en quelque sorte more geometrico, d'une théorie donnée. La théorie concernée devait donc être considérée comme produite au préalable, et les conditions prévalant à sa découverte - et pouvant expliquer sa genèse - ont été considérées comme ne jouant aucun rôle dans ce qui pouvait en justifier l'acceptabilité. C'est donc de façon explicite que le M.E.S. a prétendu qu'une théorie peut être logiquement valide et empiriquement confirmée, peu importent les voies par lesquelles on en est venu à penser le contenu logico-empirique qu'elle met en forme: il ne saurait y avoir ici ni science bourgeoise ni science prolétarienne si l'on veut dire par là que l'origine sociale (ou psychologique) des idées contenues dans les diverses théories scientifiques peut en affecter la validité logique et empirique. Cette thèse est centrale dans ce que nous avons appelé le Modèle Épistémologique Standard, et c'est en s'attaquant aux présupposés et conséquences divers de cette thèse que l'épistémologie comme discipline a le plus évolué au cours des derniers vingt ans ${ }^{41}$.

40. Kuhn (1962) et Hanson (1958) sont, respectivement, les représentants principaux de ces deux nouvelles écoles. Ceux qui douteraient du sérieux de cette "tendance " épistémologique garderont en mémoire que Simon (1977) en est le chef de file et qu'il a reçu le prix Nobel d'économie en 1979.

41. On a dit que le M.E.S. véhiculait une "statement view " de la science, une conception purement logico-linguistique. L'autre vision des choses est qualifiée tout simplement de "non statement view ". Deux entreprises s'affrontent ici, prétendant parfois, mais à tort, s'exclure mutuellement: d'une part une logique de la science, de l'autre une psychosociologie de la recherche. 


\section{4. Éléments de la crise épistémologique contemporaine.}

Tels sont les deux lieux théoriques où l'épistémologie contemporaine formule la question de la preuve en science. Cet exposé a cependant laissé deviner que de nombreux développements se sont faits jour au cours des vingt dernières années, et qui concernent aussi bien la corroboration que l'axiomatisation des théories. Ces développements ont induit un état de crise en épistémologie, et les trois obstacles majeurs qui vont être maintenant identifiés constituent pour plusieurs autant d'impasses véritables.

D'abord et avant tout l'unité de la science fait problème. Non pas tant comme situation de fait que comme exigence de principe. Le M.E.S. se voulait et se veut toujours unitaire et général, c'est-à-dire valable a priori pour toute science et toute théorie acceptable. Or, non seulement nous faut-il nous rendre compte que toutes les théories scientifiques « dignes de ce nom » ne sont pas développées au même point, mais il n'est pas certain qu'elles aient toutes à se développer de la même manière, ou qu'il leur soit possible d'atteindre au même idéal épistémologique, ou même qu'il soit souhaitable qu'elles y tendent toutes. Par exemple, suite à la proposition du modèle de la loi de couverture, un grand débat s'est ouvert, opposant au départ Hempel et Dray, sur la recevabilité de ce modèle d'explication en histoire, et ce débat n'est toujours pas $\operatorname{clos}^{42}$. Mais la question n'est pas tant celle de savoir s'il nous faut renoncer à notre modèle épistémologique unitariste que celle de savoir s'il nous faut circonscrire un endroit théorique où, certains dénominateurs communs entre toutes les sciences étant posés, il sera possible à tous les chercheurs de parler le même langage, ce qui semblera à tout le monde la condition sine qua non de l'intercompréhension et du consensus. Que l'on abandonne ou non le M.E.S., il nous reste à conserver l'idéal d'interdisciplinarité qui y fut mis en place.

Mais fait également problème la vérité de la science, car il ne nous est plus loisible de prétendre que nos théories sont vraies si nous avons ici en vue quelque "adéquation de la

42. Cf. note 30. Sur cette litigieuse question, on consultera aussi Nagel (1961, chapitre 15). 
chose et de l'entendement ». Certes, nous l'avons vu, la proposition énonçant une loi n'est jamais vérifiable, elle est tout au plus testable : nos théories scientifiques peuvent être, au mieux, affectées d'un certain degré de confirmation, et dans un contexte de compétition interthéorique, on peut avoir avantage à substituer au concept classique de vérité celui de vérisimilitude $^{43}$, concept lié à la nécessité où l'on serait de comparer entre elles les théories disponibles et à choisir, selon des critères métascientifiques, la meilleure du moment. Ici s'affrontent les conceptions réaliste et instrumentaliste de la science : le réaliste exige que puissent être déclarés "vrais" aussi bien les énoncés théoriques que les énoncés observationnels, alors que l'instrumentaliste se contente d'exiger que la théorie soit adéquate, c'est-à-dire que seuls les énoncés d'observation aient à être "vrais». L'instrumentalisme semble permettre d'introduire comme bon nous semble tous les termes théoriques nécessaires à la formulation d'une théorie qui autorisera la prédiction de phénomènes observables, peu importe que ces termes désignent ou non quelque chose de réel. Le réalisme ne se satisfait pas de ce qu'une théorie " marche", il veut pouvoir la déclarer vraie. On pourrait aisément se convaincre que l'issue de ce débat tire à conséquence en épistémologie aussi bien qu'en science même.

Le progrès de la science est aussi en question. Le problème vient de ce qu'on a de plus en plus tendance à abandonner la façon dont le M.E.S. a permis que l'on comprenne le développement historique de la science. Le concept de progrès de la science ici mis de l'avant a été celui de la réduction interthéorique: entendons par là la possibilité de rattacher une première théorie à une autre de manière qu'elle en soit déductible. Une telle réduction pouvait facilement se concevoir comme un idéal épistémologique supplémentaire puisqu'elle se trouvait à ajouter à la preuve de la validité d'une théorie donnée ${ }^{44}$. En ce sens, par exemple, on a pu considérer que la réduction des lois de Kepler aux lois de la dynamique de Newton, - c'est-à-dire, entre autres, la dérivation déductive de la forme elliptique des orbites planétaires à partir des

43. Cf. Popper (1962), chapitre 10.

44. Pour une bonne présentation de ce concept, of. Nickles (1973). 
équations différentielles définissant le champ gravitationnel constituait une preuve magistrale des lois de Kepler ${ }^{45}$. La procédure de la réduction semblait pouvoir également s'appliquer pour assurer l'unification interdisciplinaire, c'est-à-dire l'organisation déductivement subordonnée de théories traitant d'objets hétérogènes : on a cru possible une traduction sans trahison de la chimie dans le langage de la physique, de la biologie dans celui de la chimie, de la psychologie dans celui de la biologie, de la sociologie dans celui de la psychologie, et ainsi de suite. Un tel projet fut manifestement nourri par Carnap (1928) et se trouve au fondement même de cette grandiose entreprise qui se présenta comme l'« Encyclopédie de la science unifiée ${ }^{46}$.

Tout, dans ce modèle de développement, semble aujourd'hui remis en question. D'abord, le primat de la physique comme rẹine des sciences est fortement contesté et il n'est plus possible, semble-t-il, de faire accepter l'idée que la physique fournit un langage universel et premier. La spécificité des disciplines semble fonder tous les motifs de résistance. Du mème coup, la problématique du langage universel s'est trouvée entièrement transformée et constitue maintenant un champ de recherche sui generis de la sémantique formelle. Pour ce qui concerne la sémantique du langage de la science comme telle, l'intertraductibilité des théories a fait place à la question de leur incommensurabilité, compliquant d'autant la possibilité de procéder à leur comparaison et semblant nier la possibilité de comprendre le développement historique des sciences comme une série de décisions rationnelles en faveur des théories les mieux testées ${ }^{47}$.

L'interprétation réductionniste du développement des sciences permettait aussi que l'on conçoive de façon conti-

45. Considérée par Hempel (1965, chap. 10) dans le texte de 1948 où se trouve proposé le modèle de la loi de couverture, cette réduction se trouve mise en cause par Nagel (1961, chap. 4).

46. C'est Otto Neurath, un des signataires et auteur principal du manifeste du Cercle de Vienne (1929) qui fut l'instigateur de l'International Encyclopedia of Unified Science. Éditeur en chef de cette publication, il fut assisté par Rudolf Carnap et Charles Morris. L'entreprise ne fut cependant jamais achevée. Les dix-neuf monographies complétées ont été réunies en deux volumes sous le titre Foundations of The Unity of Science (Chicago : The University of Chicago Press, 1955).

47. Cette question est le legs de Kuhn (1962). 
nuiste l'histoire des sciences, chaque science étant vue comme une entreprise cumulative collectionnant ses succès expérimentaux. En effet, suivant ce modèle, une science établit des théories qui, lorsqu'elles ont atteint un haut degré de corroboration, sont acceptées par les diverses communautés scientifiques et deviennent ainsi, peu à peu, hors de la portée d'une réfutation ou d'une abrogation. L'histoire des sciences est ici dite progressiste parce que cette histoire est le fruit de l'extension de théories relativement confirmées pour certains phénomènes à d'autres domaines de l'expérience, ainsi que de l'intégration des théories relativement confirmées à des théories plus générales et fondamentales. Cette conception continuiste du progrès de la science est aujourd'hui battue en brèche. Il serait faux de prétendre qu'en fait, une théorie qui échoue à expliquer un phénomène qu'il lui revient d'expliquer est automatiquement rejetée. Le mécanisme du remplacement des théories serait beaucoup plus complexe et nécessiterait la construction de nouveaux concepts épistémologiques, tel celui de paradigme ${ }^{48}$. Dès lors, l'histoire des sciences ne serait plus conçue en termes d'évolution mais de révolutions, c'est-à-dire comme une suite de mutations affectant aussi bien les objets sur lesquels porte la théorie que le genre de questions permises et les types de solution acceptés. Ainsi donc, l'idée selon laquelle une théorie, passé un certain point de non-retour, finirait par s'incorporer à un système théorique déjà sédimenté, masquerait la dimension polémique et conflictuelle qui serait pourtant caractéristique de toute l'histoire des sciences et que seule une étude psychosociologique des communautés de chercheurs permettrait de dévoiler.

Ces dernières critiques que l'épistémologie s'adresse à elle-même prennent pour acquis que le M.E.S. cherchait avant tout à bien décrire la réalité de la science. Les partisans de ce

48. La notion de paradigme a été introduite par Kuhn (1962) et clarifiée par Kuhn (1969). Suivant cette dernière version, un paradigme est essentiellement une matrice disciplinaire (c'est-à-dire un ensemble ordonné de généralisations symboliques, de modèles ontologiques et heuristiques et de valeurs) et un ensemble d'exemples communs (c'est-à-dire de performances réussies). 
modèle répliquent habituellement à leurs détracteurs que leur entreprise est normative, et donc qu'ils cherchent non pas à rendre compte de la science pratiquée mais à représenter la science idéale. L'épistémologue féru d'histoire des sciences répond alors que le M.E.S. hypostasie la science dans ses résultats les plus admirables, peut-être, mais ne se préoccupe pas d'en expliquer la genèse historique réelle. Ce qui est en cause dans cette discussion est rien moins que la tâche de l'épistémologie elle-même. Celle que le M.E.S. s'était assignée consistait à fournir la formulation canonique la meilleure possible de ce qui pouvait compter comme science. Quelles que soient les déficiences et les insuffisances de ce modèle, son importance se mesure aisément à la place qu'il occupe dans les diatribes d'aujourd'hui, place centrale s'il en est une.

Département de philosophie Université du Québec à Montréal 


\section{BIBLIOGRAPHIE}

BLANCHE, Robert (1955). L'axiomatique. Paris, Presses Universitaires de France, coll. Initiation philosophique (4e éd. 1967).

CARNAP, Rudolf (1928). Der Logische Aufbau der Welt. Berlin. Traduction anglaise de Rolf A. George sous le titre The Logical Structure of the World. Berkeley et Los Angeles, Univ. of California Press, 1969.

CARNAP, Rudolf (1934). Logische Syntax der Sprache. Vienne. Traduction anglaise d'Amethe Smeaton sous le titre Logical Syntax of Language. Londres : Routledge \& Kegan Paul (1937).

CARNAP, Rudolf (1936-7). "Testability and Meaning ", Pbilosopby of Science 3 : 420-68; 4 : 1-40; Réédité comme monographie à New Haven (Conn.) : Whitelock's Inc., 1950.

CARNAP, Rudolf (1950). Logical Foundations of Probability. Chicago: University of Chicago Press ( $2^{\mathrm{e}}$ édition revue : 1962).

CARNAP, Rudolf (1956). "The Methodological Character of Theoretical Concepts ", dans H. Feigl et al., Eds (1956), p. 38-76.

CRAIG, William (1953). "On Axiomatizability within a System », Journal of Symbolic Logic 18 : 30-2.

CRAIG, WILLIAM (1956). "Replacement of Auxiliary Expressions ", Philosophical Review 65 : 38-55.

DRAY, William (1957). Laws and Explanation in History. Oxford : Oxford University Press.

DUHEM, Pierre M. (1906). La théorie pbysique: son objet, sa structure. Paris : Marcel Rivière.

FEIGL, Herbert et al. (Eds), (1956). Minnesota Studies in the Pbilosophy of Science, vol. I. Minneapolis : University of Minnesota Press.

FEIGL, Herbert et al. (1958). Minnesota Studies in the Pbilosophy of Science vol. II. Minneapolis : University of Minnesota Press.

FEIGL, Herbert et al. (1962). Minnesota Studies in the Philosophy of Science vol. III. Minneapolis: University of Minnesota Press.

FEIGL, Herbert (1970). "The 'Orthodox' View of Theories : Remarks in Defense as Well as Critique », dans M. Radner \& S. Winokur (1970), 3-16.

FREGE, Gottlob (1879). Begriffsschrift, eine der arithmetischen nachgebildete Formelsprache des reinen Denkens. Halle : L. Nebert. Traduction anglaise publiée dans $A$ Source book in mathematical logic. Harvard, 1967.

FREGE, Gottlob (1884). Grundlagen der Aritbmetik. Breslau : Max und Hermann Marcus. Traduction française de Claude Imbert sous le titre Les fondements de l'aritbmétique. Paris : Seuil (1969).

GAUTHIER, Yvon (1978). Méthodes et concepts de la logique formelle. Montréal : Les Presses de l'Université de Montréal.

GRICE, H.P. et STRAWSON, P.F. (1956). «In Defense of A Dogma », Pbilosophical Review 65: 141-58. Repris dans Jay F. Rosenberg et Charles Travis (Eds.), Readings in the Philosophy of Language. Englewood Cliffs, N.J., Prentice-Hall (1971), p. 81-94. 
HANSON, Norwood R. (1958). Patterns of Discovery. Cambridge, Mass. : Harvard University Press.

HARDING, Sandra G. (1976). Can Theories Be Refuted? Essays on the Dubem-Quine Thesis. Dordrecht/Boston : D. Reidel Publ. Co.

HEMPEL, Carl G. (1942). "The Function of General Laws in History ", The Journal of Philosophy 39:35-48 ; Texte repris, dans une version légèrement modifié in (1965), chapitre 9, p. 231-243.

HEMPEL, Carl G. (1958), "The Theoretician's Dilemma : A Study in the Logic of Theory Construction ", dans Herbert Feigl, Michael Scriven \& Grover Maxwell (Eds) (1958). Repris avec modifications dans Hempel (1965), chapitre 8, pp. 173-226.

HEMPEL, Carl G. (1965). Aspects of Scientific Explanation and Other Essays in the Pbilosopby of Science. New York: The Free Press.

HEMPEL, Carl G. (1966). Philosophy of Natural Science. Englewood Cliffs, N.J. : Prentice Hall. Traduction française sous le titre Éléments d'épistémologie. Paris : Librairie Armand Colin, 1972.

HEMPEL, Carl G. (1970). "On the 'Standard Conception' of Scientific Theories ", dans M. Radner \& S. Winokur (1970), p. 142-63.

KAN'T, Emmanuel (1781). Critique de la raison pure. T'raduction française de A. Tremesaygues et B. Pacaud, Paris : Presses Universitaires de France, 1944.

KUHN, Thomas S. (1962). The Structure of Scientific Revolutions. Chicago : University of Chicago Press. Il en existe une mauvaise traduction française, établie à partir de la seconde édition américaine revue et augmentée, publiée sans identification du traducteur sous le titre La structure des révolutions scientifiques. Paris : Flammarion, 1972.

KUHN, Thomas S. (1969). «Postscript " à la seconde édition de (1962) Cf. «Postface - 1969 » dans la traduction française de (1962).

KYBURG, Henry E. Jr. (1968). Philosopby of Science : A Formal Approach. New-York : Macmillan.

LEWIS, Clarence I. (1929). Mind and the World-Order. New-York, Chicago, et Boston : Scribners.

MORGENBESSER, Sidney (1967). Philosophy of Science Today. New York : Basic Books.

NAGEL, Ernest (1961). The Structure of Science. New-York; nouvelle édition, Indianapolis \& Cambridge: Hackett Publ. Co., 1979.

NAGEL, Ernest, SUPPES, Patrick et TARSKI, Alfred (Eds) (1962). Logic, Methodology and Philosophy of Science: Proceedings of the 1960 International Congress. Stanford : Stanford University Press.

NICKLES, Thomas (1973). "Two Concepts of Intertheoretic Reduction ", Journal of Philosophy 70 : 181-201.

POINCARE, Henri (1902). La Science et l'bypothèse. Paris : Flammarion.

POPPER, Karl R. (1944-5). Poverty of Historicism. Il s'agit de trois articles parus dans la revue Economica. On en trouve la traduction française sous le titre Misère de l'bistoricisme. Paris : Plon, 1956. 
POPPER, Karl R. (1959). The Logic of Scientific Discovery. Londres: Hutchison. Traduction française établie par Nicole Thyssen-Rutten et Philippe Devaux, La logique de la découverte scientifique. Paris: Payot, 1973. (Une première version allemande avait déjà été publiée en 1934-5).

POPPER, Karl. R. (1962). Conjectures and Refutations. New York : Basic Books et London: Routledge \& Kegan Paul Ltd.

PUTNAM, Hilary, (1962). "What Theories are Not", dans Ernest Nagel, Patrick Suppes \& Alfred Tarski (1962) p. 240-51.

QUINE, Willard von Orman, (1951). "Two Dogmas of Empiricism ", Philosopbical Review 60: 20-43. Repris dans From a Logical Point of View, Cambridge (Mass.) : The M.I.T. Press, 1953.

QUINE, Willard von Orman (1960). Word and Object. Cambridge (Mass.) : The M.I.T. Press.

RADNER, M. et WINOKUR, S. (Eds) (1970). Minnesota Studies in the Pbilosopby of Science, vol. IV. Minneapolis : University of Minnesota Press.

REICHENBACH, Hans (1924). Axiomatik der Relativistischen Raum-ZeitLebre. Braunschweig: Vieweg. Trad. angl. : Axiomatization of the Theory of Relativity. Berkeley : University of California Press, 1969.

ROBERT, Serge (1978). La logique, son bistoire, ses fondements. Montréal : Éditions Le Préambule, Collection Science et Théorie.

RUSSELL, Bertrand et WHITEHEAD, Alfred N. (1910-3). Principia Matbematica, 3 vol. Cambridge : Cambridge University Press.

RUSSELL, Bertrand (1918). "The philosophy of Logical Atomism ", The Monist 28 : 495-527 ; 29 (1919) : 32-63, 190-222 et 345-380. Repris dans Russell (1956).

RUSSELL, Bertrand (1956). Logic and Knowledge. R.C. Marsh, (Ed.), London : Allen \& Unwin.

SCHAFFNER, Kenneth F. (1969). «Correspondance Rules », Pbilosopby of Science $36: 280-90$.

SIMON, Herbert A. (1977). Models of Discovery and Other Topics in the Metbods of Science. Dordrecht/Boston : D. Reidel Publ. Co.

SUPPE, Frederick (1974). «The Search for Philosophic Understanding of Scientific Theories», dans F. Suppe (Ed.) The Structure of Scientific Theories. Urbana-Chicago-London: University of Illinois Press, 1974, pp. 1-241. La seconde édition (1977) a été notamment augmentée d'une postface de Suppe qui vient compléter l'exposé de 1974.

SUPPES, Patrick (1962). « Models of Data », dans E. Nagel, P. Suppes \& A. Tarski (Eds) (1962) p. 252-61.

SUPPES, Patrick (1967). «What Is a Scientific Theory?», dans S. Morgenbesser (1967), p. 55-67.

SUPPES, Patrick (1968). "The Desirability of Formalization in Science ", Journal of Philosophy $65:$ 651-64. 
TARSKI, Alfred (1944). "The Semantic Conception of Truth », Pbilosophy and Phenomenological Research $4: 341-75$. On en trouvera la traduction française dans le recueil de Tarski intitulé Logique, sémantique, métamathématique 1923-44. Paris : Armand Colin, 1974, tome 2, pp. 265-305.

WITTGENSTEIN, Ludwig (1921). Logisch - pbilosophische Abbandlung dans Wilhelm Ostwald (Ed.), Annalen der Naturphilosophie. Republié avec, en parallèle, une traduction anglaise de C.K. Ogden et F.P. Ramsey, et une Introduction de Bertrand Russell, sous le titre Tractatus Logico-Pbilosopbicus. Londres: Routledge \& Kegan Paul (1962). Traduction française de Pierre Klossowski, Paris : Gallimard (1931). Nouvelle traduction anglaise de D.F. Pears et B.F. McGuinness, Londres : Routledge \& Kegan Paul, New York: Humanities Press (1961). 